This item was submitted to Loughborough's Institutional Repository (https://dspace.lboro.ac.uk/) by the author and is made available under the following Creative Commons Licence conditions.

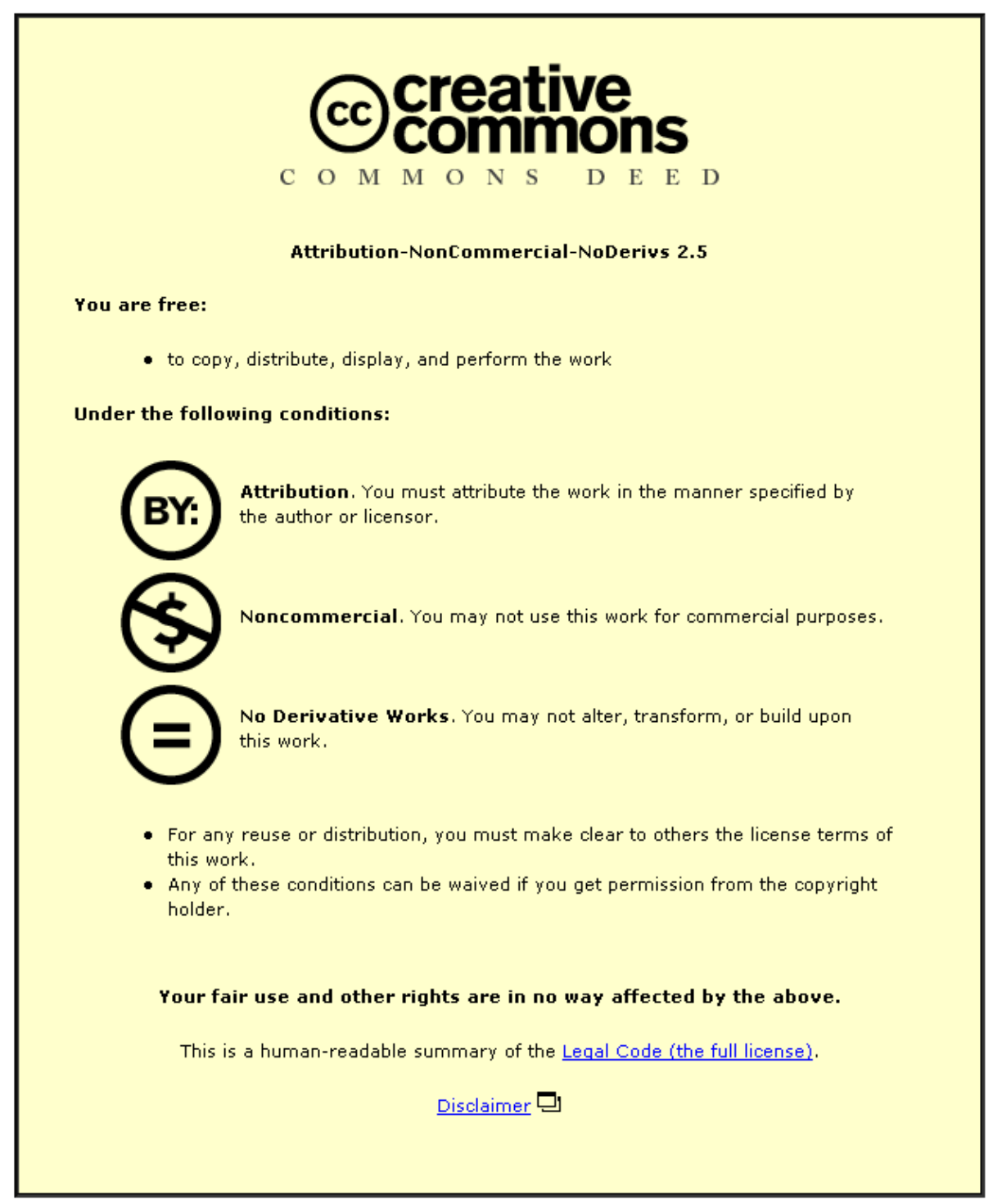

For the full text of this licence, please go to: http://creativecommons.org/licenses/by-nc-nd/2.5/ 


\title{
THE LIMITS OF AERIAL TWISTING TECHNIQUES IN THE AERIALS EVENT OF FREESTYLE SKIING
}

\author{
M.R. Yeadon \\ School of Sport, Exercise and Health Sciences, Loughborough University, LE11 3TU, UK
}

\begin{abstract}
In the aerials event of freestyle skiing, athletes perform three somersaults with up to five twists. This study investigated the twisting limits of such movements using a computer simulation model of aerial movement. The abilities of various asymmetrical arm and hip techniques to produce twist during flight were investigated using 10 simulations to maximise twist and allow reorientation prior to landing. It was found that 4,5 , or 6 twists could be produced during three somersaults. The main limiting factor was the increased whole body frontal moment of inertia due to the equipment which restricted the amount of tilt resulting from an asymmetrical arm movement. It was concluded that reductions in equipment mass might make such movements easier to achieve but would be unlikely to allow advances beyond the limits found.
\end{abstract}

Keywords: computer simulation, aerials, asymmetrical arms

\section{Introduction}

The aerial phase of a sports movement may be relatively simple as in running, for example, in which the body has a small amount of angular momentum which allows forwards rotation of the body during flight from the takeoff on one leg to the landing on the other leg. In some sports the aerial movements are complex and form the basis of the sport, as in springboard and platform diving, trampolining, tumbling, gymnastics dismounts and the aerials event of freestyle skiing. Typically points are awarded for difficulty (movement complexity) and performance (aesthetics). Currently the most complex movements competed in freestyle aerials are triple somersaults with five twists.

In the aerials event the athlete skis down an inclined in-run to gather speed and enters a curved takeoff ramp or "kicker" which launches the aerialist on a suitable flight path with appropriate angular momentum. After completing the intended somersault and twist rotations the aerialist lands on an inclined slope (Figure 1). As twist is completed at the end of each somersault the arms are spread wide (abducted) to show separation of these phases. Typically there is only up to $1 \frac{1}{2}$ twists in each of the first and third somersaults since the first somersault comprises only $\frac{3}{4}$ of a revolution and the third somersault is used for landing preparation. In the second somersault there may be as many as three twists.

Twist may be initiated during contact with the kicker (contact twist) or may be produced after takeoff (aerial twist) as found by Yeadon (1989). One problem associated with contact twist is that the skier is prone to be tilted laterally on landing (Yeadon, 1993b) which can lead to higher forces on one leg and difficulty in controlling the landing. Aerial twisting techniques do not suffer inherently from this problem although body orientation prior to landing still needs to be controlled. Aerial twist may be produced by using asymmetrical arm or hip movements to produce tilt during flight (Yeadon, 1989, 1993c), typically during the first somersault. The aim of this study is to investigate how much twist can be achieved using various aerial twisting techniques.

\section{Methods}

A computer simulation model of aerial movement (Yeadon et al., 1990) was used to determine the limits of various The aerial phase of a sports movement may be relatively simple as in running, for example, 


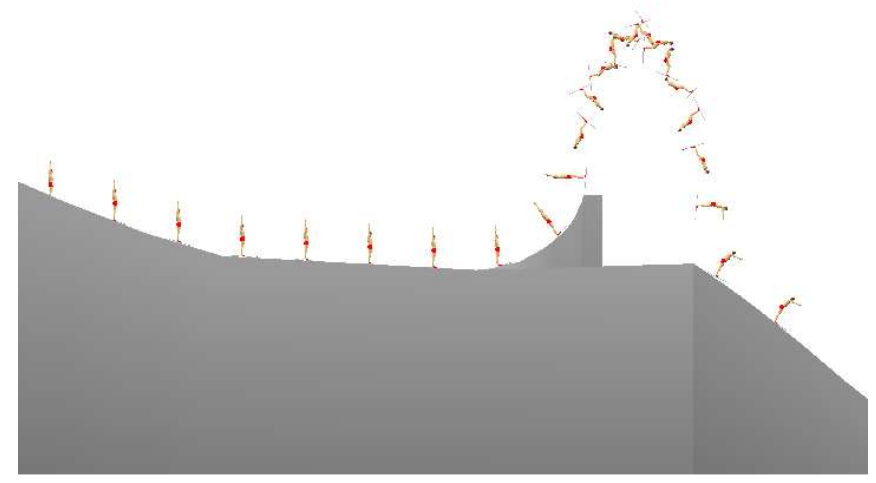

Figure 1: In-run, takeoff, flight and landing phases.

in which the body has a small amount of angular momentum which allows forwards rotation of the body during flight from the takeoff on one leg to the landing on the other leg. In some sports the aerial movements are complex and form the basis of the sport, as in springboard and platform diving, trampolining, tumbling, gymnastics dismounts and the aerials event of freestyle skiing. Typically points are awarded for difficulty (movement complexity) and performance (aesthetics). Currently the most complex movements competed in freestyle aerials are triple somersaults with five twists.

In the aerials event the athlete skis down an inclined in-run to gather speed and enters a curved takeoff ramp or "kicker" which launches the aerialist on a suitable flight path with appropriate angular momentum. After completing the intended somersault and twist rotations the aerialist lands on an inclined slope (Figure 1). As twist is completed at the end of each somersault the arms are spread wide (abducted) to show separation of these phases. Typically there is only up to $1 \frac{1}{2}$ twists in each of the first and third somersaults since the first somersault comprises only $\frac{3}{4}$ of a revolution and the third somersault is used for landing preparation. In the second somersault there may be as many as three twists.

Twist may be initiated during contact with the kicker (contact twist) or may be produced after takeoff (aerial twist) as found by Yeadon (1989). One problem associated with contact twist is that the skier is prone to be tilted laterally on landing asymmetrical arm and hip techniques for producing aerial twist. The original model comprised 11 segments and required the initial angular momentum and body orientation as input together with the time histories of the joint angles. In this application movement was restricted to five joint centres with two controlled degrees of freedom at each joint so that the model effectively comprised six segments (Figure 2). Side flexion was shared between the hips and the spine (Figure 2) as was hyperextension whereas forward flexion occurred solely at the hip joints (Yeadon, 1990a). In addition the two legs moved together so that the six degrees of freedom at the hip joints and spine became effectively two independent degrees of freedom. Constant angular momentum during flight was assumed and the equation of motion was solved numerically for whole body angular velocity from which somersault, tilt and twist angles (the three uncontrolled degrees of freedom) were obtained by numerical integration (Yeadon et al., 1990). Somersault gave the whole body rotation about the (horizontal) angular momentum vector, tilt gave the angle between the longitudinal axis and the vertical plane perpendicular to the angular momentum vector, and twist gave the rotation about the longitudinal axis. The model was evaluated previously by comparing the twist angles from simulation with six performances at the 1988 Winter Olympic Games: differences were less than 0.08 revolutions of twist (Yeadon, 1989). It was assumed that the effects of aerodynamic drag forces on the rotational movement could be neglected.

Anthropometric measurements of a Swiss aerials team member wearing a ski suit were taken, having obtained informed consent as approved by Loughborough University Ethical Advisory Committee. The measurements were input to the inertia model of Yeadon (1990b) together with the segmental densities of Chandler et al. (1975) to calculate segmental inertia parameters. A density scaling factor was used for body segments covered by the suit to adjust segmental densities so that the whole body mass matched the value as measured with weighing scales. Subsequently the densities of the head, hands and feet were 


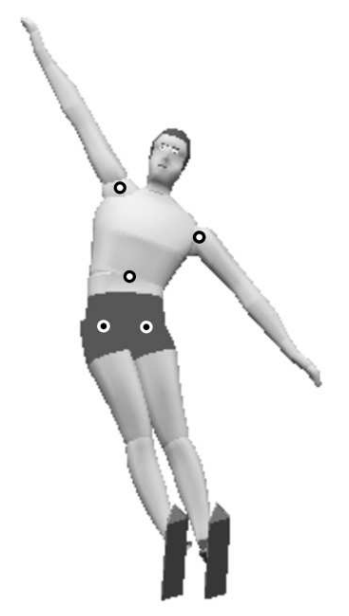

Figure 2: Six segment model of aerial movement with two degrees of freedom at each of the five joints.

each adjusted to account for the additional mass of helmet, gloves and boots. Inertia parameters of the bindings and skis were calculated from mass and length measurements and the lower leg inertias were adjusted to take these into account (Table 1).

Table 1: Segmental inertia parameters of an aerialist

\begin{tabular}{|l|c|c|c|c|c|c|}
\hline segment & mass $(\mathrm{kg})$ & length $(\mathrm{m})$ & CoM $(\mathrm{m})$ & $\mathrm{Ix}\left(\mathrm{kg} \cdot \mathrm{m}^{2}\right)$ & $\mathrm{Iy}\left(\mathrm{kg} \cdot \mathrm{m}^{2}\right)$ & $\mathrm{Iz}\left(\mathrm{kg} \cdot \mathrm{m}^{2}\right)$ \\
\hline pelvis & 9.17 & 0.21 & 0.10 & 0.07 & 0.09 & 0.10 \\
\hline thigh & 8.93 & 0.45 & 0.19 & 0.16 & 0.16 & 0.04 \\
\hline shank & 9.43 & 0.50 & 0.38 & 0.55 & 0.29 & 0.27 \\
\hline abdomen & 8.17 & 0.30 & 0.09 & 0.06 & 0.07 & 0.09 \\
\hline chest & 18.61 & 0.19 & 0.07 & 0.43 & 0.45 & 0.19 \\
\hline arm & 2.30 & 0.30 & 0.13 & 0.02 & 0.02 & 0.00 \\
\hline forearm & 1.68 & 0.36 & 0.18 & 0.03 & 0.03 & 0.00 \\
\hline
\end{tabular}

Note: CoM is the distance of segment mass centre from proximal joint; Ix, Iy, Iz are moments of inertia about lateral, frontal and longitudinal axes through segment mass centre

Various sequences of asymmetrical arm and hip movements were used to produce tilt away from the vertical somersault plane during an aerial simulation using the model of Yeadon et al. (1990). Each change in joint angle was specified by the start and end angle values and the start and end times and was effected using a quintic function (Figure 3) with zero velocity and acceleration at the endpoints (Hiley and Yeadon, 2003). For an arm movement through $180^{\circ}$ a minimum duration of $0.30 \mathrm{~s}$ was imposed based on observation of competition recordings. A minimum duration of $0.20 \mathrm{~s}$ was also imposed on a side flexion movement through $40^{\circ}$ (angle between legs and upper trunk).

In order to maximise the amount of twist produced by each technique, the timing of the arm and hip movements were adjusted to maximise the amount of tilt achieved in the first somersault. In the second somersault the arms were adducted to maximise the twist and in the third somersault asymmetrical arm movements were used to reduce the tilt to near zero. Adjustments were made to the initial (somersault) angular momentum to compensate for the various body configurations and allow the movements to be performed with the body extended throughout the twisting phases. Prior to landing the hips were flexed and the arms brought in front to provide an appropriate landing configuration.

After the completion of the first and second somersault the arms were abducted to show separation of the three phases of twist. The following constraints were followed when producing a simulation: (a) at the 1.0 and 2.0 somersault positions the twist was within 0.25 revolutions of the identified whole number 


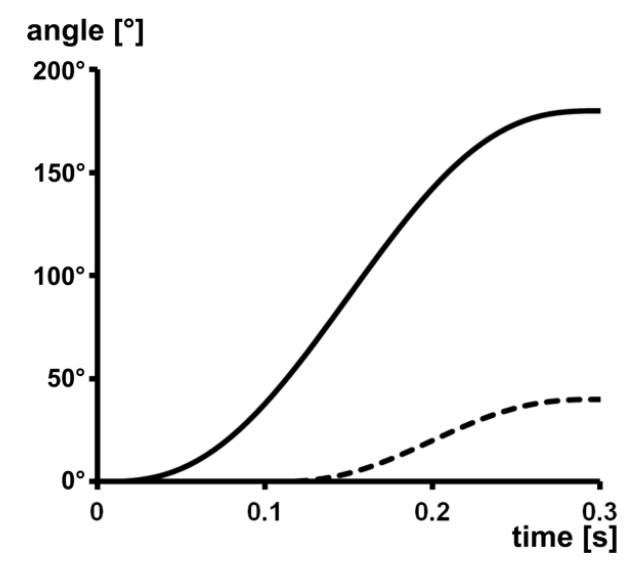

Figure 3: Change of arm angle from 0 to $180^{\circ}$ from time $0.0 \mathrm{~s}$ to time $0.3 \mathrm{~s}$ (solid line) and change of side flexion from 0 to $40^{\circ}$ from time $0.1 \mathrm{~s}$ to time $0.3 \mathrm{~s}$ (dashed line) using a quintic function with zero velocity and acceleration at the endpoints.

of half twists, (b) the identified number of twists was reached within $\pm 30^{\circ}$ of vertical around 1 and 2 somersaults, (c) the amount of symmetrical arm abduction separating the phases was at least $30^{\circ}$ and was shown as the identified twist was reached, (d) the final tilt, twist and somersault angles were within $2^{\circ}$ of target, (e) the time of flight was $2.9 \mathrm{~s}$. In order to maximise the twist and to comply with these constraints, a thorough manual search was conducted using timing steps of $0.01 \mathrm{~s}$ when generating each simulation.

The search procedure comprised: (a) adjusting the start and end times of the initial arm angle changes through $180^{\circ}$ or side flexion through $40^{\circ}$ in order to maximise the tilt angle, followed by (b) adjustment of the timing of the symmetrical arm abduction to complete 1.0 or 1.5 twists close to the completion of the first somersault (body vertical), (c) timing of the subsequent symmetrical arm adduction to give a fixed arm abduction angle in the middle somersault, (d) adjustment of this symmetrical arm abduction angle to give an integral number of half twists at the second somersault position, (e) timing and amount of subsequent arm abduction at the second somersault position to produce 1.0 or 1.5 twists in the third somersault, (f) timing of the sequential arm movements to remove the tilt and complete the integral number of twists, (g) adjustment of the initial angular momentum to obtain target somersault rotation at the same time as target twist and tilt angles. While much of the above search procedure was sequential some parts were interactive and required fine tuning to produce final orientation angles within $2^{\circ}$ of target. Each optimisation of a simulated technique took several hours to complete.

It should be noted that the constraints used in (b) are more stringent than the $\pm 90^{\circ}$ currently in practice (FIS, 2010) in order to avoid ambiguity on how a given movement should be named. A consequence of this is that simulations of aerial twist cannot produce two twists in the first somersault.

Ten simulations employing asymmetrical arm and hip movements were produced (Figure 4). The arm and hip movements used in the first somersault to produce tilt comprised:

A: left arm down the side followed by right arm down the front at around a quarter twist

B: left arm down the side followed by right arm down the side at around a half twist

C: right arm down the front then double arm switch followed by right arm down the front

D: left arm down side followed by double arm switch at around a half twist

E: left down side, right down front at a quarter twist, left up side at half twist

F: starting with right arm down: double arm switch, right down front at a quarter twist

G: starting with right arm down: double arm switch, right down side at a half twist

H: $40^{\circ}$ side flexion with wide arms followed by body extension and arm adduction

I: $40^{\circ}$ side flexion and left arm down side followed by right arm down side at a half twist

$\mathrm{J}: 40^{\circ}$ side flexion followed by both arms down front around a quarter twist

This list includes the major aerial twisting techniques currently used in international competition.

In each simulation the tilt was removed in the final somersault using: left arm up the side (abduction) and right arm down the side (adduction) at a half twist remaining, followed by right arm up the side as the twist was completed. 


\section{Results}

The 10 simulations using asymmetrical arm and hip movements produced tilt angles ranging from $8.7^{\circ}$ to $15.2^{\circ}$ (Figure 4) resulting in 4,5 or 6 twists in the three somersaults (Table 2). The durations of these asymmetrical movements (Figure 4) ranged from $0.5 \mathrm{~s}$ to $0.7 \mathrm{~s}$ with the longer times corresponding to the more complex movements. The arm abduction angles in the middle somersault ranged from $3^{\circ}$ to $15^{\circ}$ indicating the extent to which adjustments could be made to compensate in performances if the twist rate was slower than in the simulation.

Table 2: Simulations of aerial twist produced by asymmetrical arm and hip movements

\begin{tabular}{|c|l|l|c|c|r|}
\hline simulation & description & outcome & twists & tilt & arms \\
\hline A & LDS, RDF & $1-2-1$ & 4 & $8.7^{\circ}$ & $3^{\circ}$ \\
\hline B & LDS, RDS & $1-2-1$ & 4 & $9.9^{\circ}$ & $11^{\circ}$ \\
\hline C & RDF, DAS, RDF & $1-3-1$ & 5 & $13.4^{\circ}$ & $5^{\circ}$ \\
\hline D & LDS, DAS & $1-3 \frac{1}{2}-1 \frac{1}{2}$ & 6 & $15.2^{\circ}$ & $175^{\circ}$ \\
\hline E & LDS, RDF, LUS & $1-3 \frac{1}{2}-1 \frac{1}{2}$ & 6 & $14.6^{\circ}$ & $175^{\circ}$ \\
\hline F & AS, DAS, RDF & $1 \frac{1}{2}-2 \frac{1}{2}-1$ & 5 & $12.2^{\circ}$ & $9^{\circ}$ \\
\hline G & AS, DAS, RDS & $1 \frac{1}{2}-3-1 \frac{1}{2}$ & 6 & $13.9^{\circ}$ & $9^{\circ}$ \\
\hline H & side flexion & $1-2-1$ & 4 & $10.4^{\circ}$ & $15^{\circ}$ \\
\hline I & side, asy-arms & $1-2 \frac{1}{2}-1 \frac{1}{2}$ & 5 & $12.5^{\circ}$ & $15^{\circ}$ \\
\hline J & side, LRDF & $1-3-1$ & 5 & $12.2^{\circ}$ & $9^{\circ}$ \\
\hline
\end{tabular}

Note: $\mathrm{LDS}=$ left arm down side (arm adduction)

$\mathrm{RDF}=$ right arm down front (arm extension)

DAS $=$ double arm switch (LDS+RUS)

LUS $=$ left arm up side (abduction)

arms $=$ symmetrical arm abduction in middle somersault

$175^{\circ}=5^{\circ}$ abduction from arms overhead

Simulations with two asymmetrical arm movements through $180^{\circ}$ produced between $8.7^{\circ}$ and $9.9^{\circ}$ of tilt resulting in 4 twists (Figure 5: A, B) whereas simulations with three asymmetrical arm movements produced between $12.2^{\circ}$ and $15.2^{\circ}$ tilt resulting in 5 or 6 twists (Figure 5: C, D, E, F, G). Simulation $\mathrm{H}$ which used side flexion of the hips with symmetrical arms produced $10.4^{\circ}$ tilt and 4 twists (Figure 5 : $\mathrm{H}$ ) whereas the introduction of asymmetrical arms in simulation I gave $12.5^{\circ}$ tilt and 5 twists (Figure 5: I). In simulation J both arms were lowered parallel to the sagittal plane once the hip asymmetry had produced a quarter twist, giving $12.2^{\circ}$ tilt and 5 twists (Figure 5: J).

In simulations $\mathrm{A}, \mathrm{B}$ and $\mathrm{H}$ in which the tilt was $10.4^{\circ}$ or less, it was possible to remove the tilt by abducting the right arm overhead and adducting the left arm to the side at a half twist short of target, followed by abducting the left arm overhead as the total twist neared completion (Figure 6). In the remaining simulations where the tilt was $12.2^{\circ}$ or more, this arm movement was supplemented by a lowering of the right arm as the left arm was raised resulting in arm asymmetry at landing (Figure 6). The durations of these asymmetrical arm movements (Figure 6) ranged from $0.5 \mathrm{~s}$ to $0.8 \mathrm{~s}$ with the shorter times corresponding to simulations with $1 \frac{1}{2}$ twists in the third somersault (D, E, G, I).

In simulation B the somersault rate was approximately constant throughout while the twist rate was low at the start and end and the tilt angle was approximately constant between $0.7 \mathrm{~s}$ and $2.1 \mathrm{~s}$ (Figure 7a). The tilt was produced by adducting first the left arm and then the right arm from an overhead position (Figure 7b). Subsequently the arms were abducted to $30^{\circ}$ around the 1.0 somersault and 2.0 somersault positions with an arm angle of $11^{\circ}$ during the middle somersault (Figure $7 \mathrm{~b}$ ). Finally the arms were abducted sequentially to $180^{\circ}$ (overhead) around the 3.5 and 4.0 twist positions to remove the tilt prior to landing (Figure $7 \mathrm{~b}$ ). 


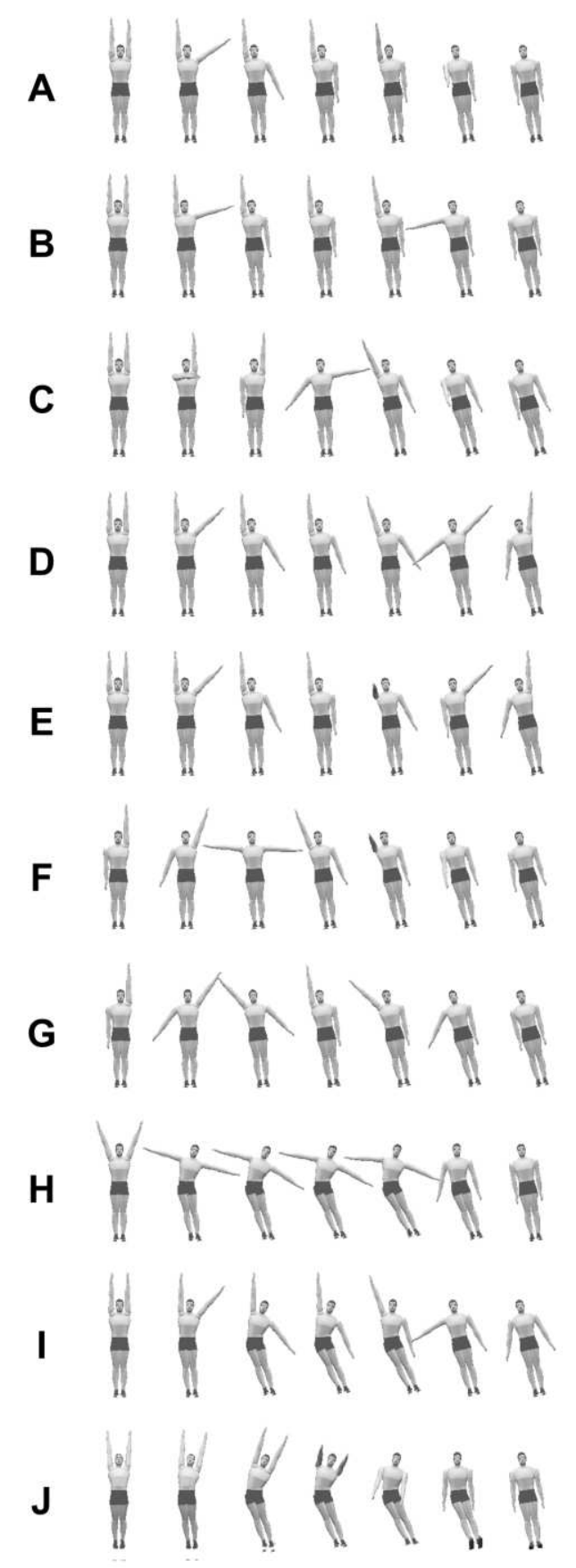

Figure 4: Asymmetrical movements and the resulting tilt in the first somersault.

(Graphics were produced from a simulation by resetting somersault and twist angles to zero.) 


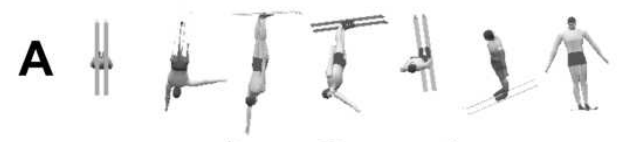

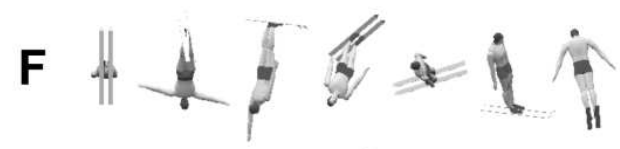

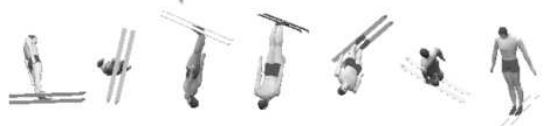
\& 4 in

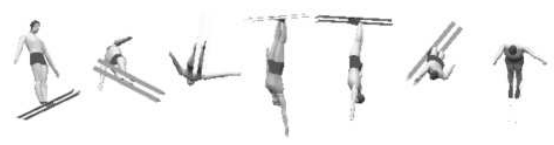

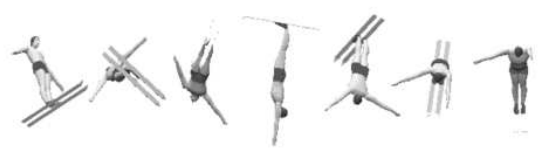
B \#小 TH
G the the

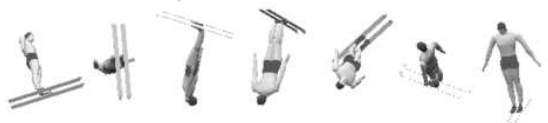
y.

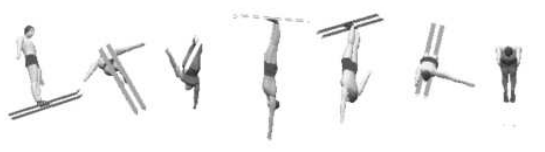

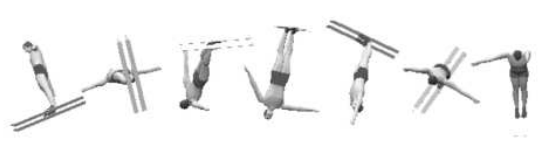
c $\#$ t $t=1$

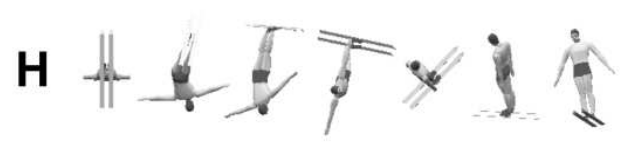

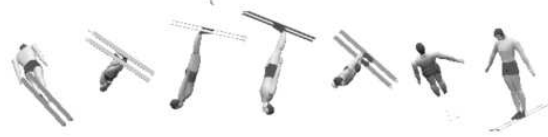

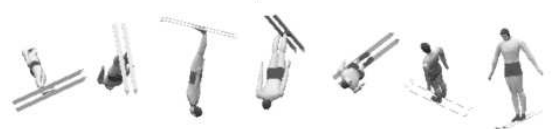

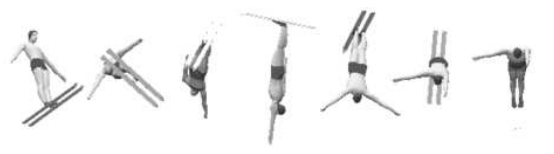

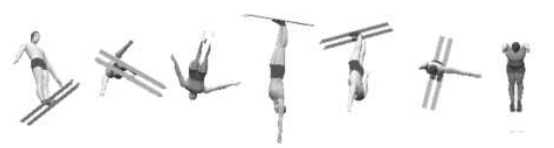
D \# R D F
1 H $k+F+1$

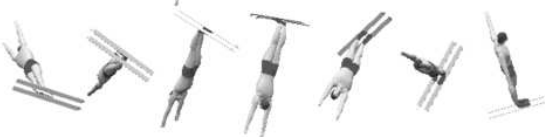

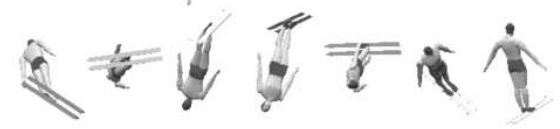
Ix⿺𠃊t)
专4
E H R F I I
J 1 I I
$y+4 A+1$

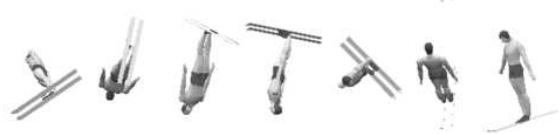

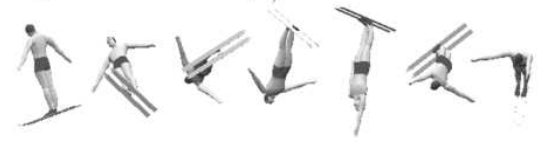

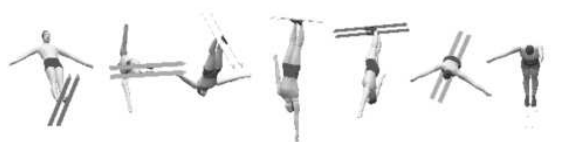

Figure 5: Graphics representations of the 10 simulations described in Table 2. 


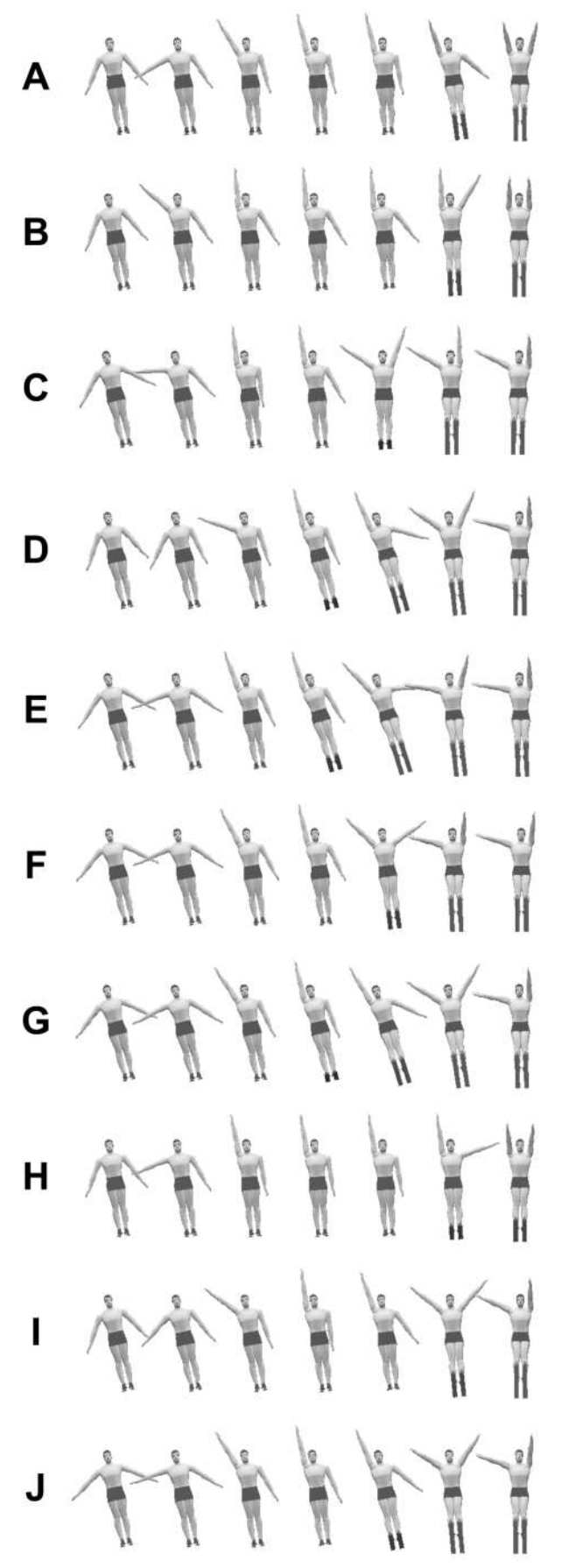

Figure 6: Removal of tilt in third somersault using asymmetrical arm movements.

(Graphics were produced from a simulation by resetting somersault and twist angles to zero.) 

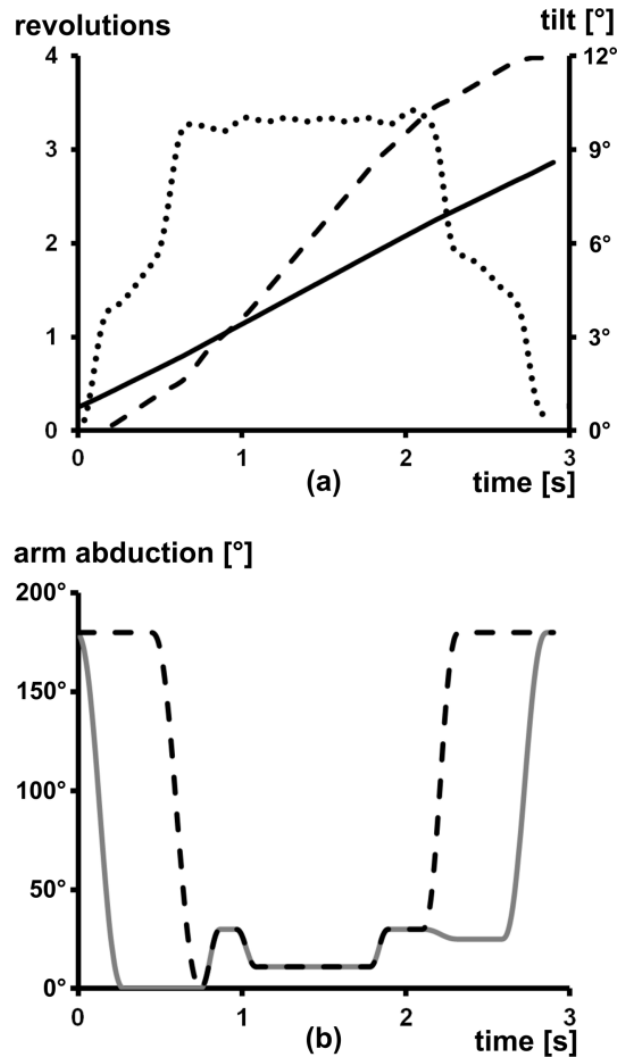

Figure 7: Angles of (a) somersault (solid line), twist (dashed line), tilt (dotted line) and (b) left arm abduction (solid grey line), right arm abduction (dashed line) in simulation B. 


\section{Discussion}

The aim of this investigation was to maximise the number of twists that can be produced in a straight triple somersault using various asymmetrical arm and hip movements to produce aerial twist. The 10 movements considered were able to produce between 4 and 6 twists depending on the complexity of the arm movements. At least half of the total twist occurred in the middle somersault. In the first somersault there is only three quarters of a somersault revolution available and the priority is to maximise tilt rather than twist. In the third somersault there is less than one somersault revolution available and the priority is to remove the tilt, adjust the final twist and somersault orientation, and adopt a suitable body configuration for landing. As a consequence the amount of twist in each of the first and third somersaults is limited to 1 or $1 \frac{1}{2}$ twists.

Minimum movement times of $0.3 \mathrm{~s}$ and $0.2 \mathrm{~s}$ were imposed on arm movements of $180^{\circ}$ and side flexion through $40^{\circ}$ based on recordings of competitive performances. A sensitivity analysis was carried out using reductions of these times by $10 \%$. Although this allowed some increase in arm abduction in the middle somersault it did not permit an additional twist.

It was assumed that the effects of aerodynamic drag on the rotations produced in the simulations could be neglected. In reality the main effect of aerodynamic drag will be to slow the somersault rate during flight producing a decline in the angular momentum about the mass centre. This decline was estimated to be $10 \%$ by averaging the times to rotate through one somersault in the first part of flight and one somersault in the second part of flight for 20 actual performances and comparing these with the equivalent average times from the 10 simulations. The effect of this decline on a movement will be minimal since the number of twists per somersault is given by $\left[\left(\mathrm{I}_{\mathrm{T}} / \mathrm{I}_{\mathrm{L}}\right)-1\right] \sin \theta$ where $\theta$ is the tilt angle (Yeadon 1993a) which will be unchanged. As a consequence the real movements would look the same as the simulated movements but with the time stretched towards the end of flight. The time of mid-flight in the simulations will have been over-estimated by around $0.04 \mathrm{~s}$.

This study was confined to movements with twist produced after takeoff whereas it is also possible to start to twist during takeoff. It is unlikely that the use of such contact twist will lead to an increase in the total amount of twist possible. This is suggested by the results of the study of Yeadon (1989) in which aerial techniques produced just as much twist as contact techniques. Current techniques use much less contact twist than those in this earlier study in which the skis themselves had considerable $\left(>30^{\circ}\right)$ twist rotation at takeoff. This is likely to be a result of current judging penalties when twisting on the kicker is observed (Federation Internationale de Ski, 2010). Additionally the use of contact twist means that it is not possible to have zero tilt away from a vertical sagittal plane as well as zero twist velocity at landing. It is quite common for landing to be heavier on one ski due to a failure to reduce the tilt sufficiently.

It is harder to twist in the aerials event compared to trampolining or diving due to the equipment (boots, bindings, skis, helmet) used in aerials. It might be thought that this greater difficulty is a consequence of the larger moment of inertia $\mathrm{I}_{\mathrm{L}}$ of the body about the longitudinal axis due to the ski length. While $\mathrm{I}_{\mathrm{L}}$ is some $65 \%$ larger, the moment of inertia $\mathrm{I}_{\mathrm{T}}$ about a transverse axis is also some $72 \%$ larger than without equipment (arms adducted). The number of twists per somersault is given by $\left[\left(\mathrm{I}_{\mathrm{T}} / \mathrm{I}_{\mathrm{L}}\right)-1\right] \sin \theta$ where $\theta$ is the tilt angle (Yeadon 1993a) and will therefore be similar in each case. The value of $\left[\left(\mathrm{I}_{\mathrm{T}} / \mathrm{I}_{\mathrm{L}}\right)-1\right]$ (with the body straight and arms adducted) using the inertia set in this study was 14.3. The segmental inertias from an earlier study (Yeadon, 1989) resulted in values ranging from $7 \%$ below this value to $8 \%$ above this value. Thus the number of twists per somersault will not vary greatly from the results of this study as a consequence of individual inertia variation.

The reason that twisting in aerials is more difficult is that it is harder to produce tilt. While the moment of inertia of an arm increases only slightly with equipment (gloves), the whole body moment of inertia about a transverse axis increases by $72 \%$ due to the mass of helmet, boots and skis. Thus whereas moving one arm through $180^{\circ}$ will produce $7.4^{\circ}$ of tilt without equipment, the same movement will only produce $4.8^{\circ}$ of tilt with equipment. As a consequence any reduction in the mass of the equipment (helmet, boots, skis) will facilitate the production of tilt and twist. On the other hand producing an additional twist $\left(360^{\circ}\right)$ in the middle somersault would require a $50 \%$ reduction in equipment mass. This suggests that the twist limits provided by the 10 simulations are unlikely to change as a result of future equipment changes.

A $180^{\circ}$ arm movement will produce $4.8^{\circ}$ of tilt when gloves are worn. Without gloves the moment of inertia of the arm is less and only $4.5^{\circ}$ of tilt will be produced by the same arm movement. This 
indicates that heavier gloves should be of advantage in producing tilt although more mass in the glove will require larger torques at the shoulder and movements may be more difficult to control. Additionally any such gain is likely to be relatively small (say $<5 \%$ ). The segmental inertias from an earlier study (Yeadon, 1989) gave rise to tilt angles ranging from $5 \%$ to $15 \%$ lower than the $4.8^{\circ}$ of tilt obtained for the inertias in this study. Thus any increases arising from individual inertia variations are again likely to be relatively small.

Those simulations (D, E) that keep the arms overhead in the middle somersault benefit from an increase in the number of twists per somersault but require more initial somersault momentum since $I_{T}$ is $21 \%$ larger than having the arms by the sides. The range of relative angular momenta of the 10 simulations was $6 \%$. Angular momentum (and time of flight) may be set appropriately for a given movement by using a faster or slower approach or modifying the ankle plantarflexion torque during contact with the kicker immediately prior to takeoff. The ability to produce consistent angular momentum for a limiting movement is important. In performances of such movements adjustments are made to compensate for too much somersault momentum by moving the arms higher up the body in the middle somersault. In two of the simulations ( $\mathrm{D}$ and $\mathrm{E}$ ) the arms were held overhead during the middle somersault. To compensate for low somersault momentum in performances of these movements the arms would be positioned lower down the body while preserving the longitudinal moment of inertia and the twist rate. For the remaining eight limiting movements, low angular momentum is problematic since the arms are already low and reducing the moment of inertia about a lateral axis by flexing at the knees and hips in order to speed up the somersault will also slow down the twist. This will result in the twist being completed after the second somersault position, leaving less time in the third somersault for the remaining twist and the removal of tilt.

In the 10 simulations the arm abduction angles ranged from $3^{\circ}$ to $15^{\circ}$ in the middle somersault. Adducting the arms by $10^{\circ}$ corresponds to a twist rate increase of $12 \%$ (the somersault rate changes by less than $0.2 \%$ ). Thus each simulation provides some flexibility for compensation in a performance where insufficient tilt has been produced. For aerialists with different segmental inertias and equipment to those used in this study there will be variation in the amount of tilt that will result from an arm movement and the number of twists per somersault that can be achieved. While the margins provided by the arm abduction angles may allow some of these aerialists to perform all of the movements shown here, some aerialists may not be able to execute all of these movements since the margins are not high. Additionally some aerialists may be able to complete the movements with greater arm abduction margins but since the margins are not high there is little scope for an additional $\left(360^{\circ}\right)$ twist. As a consequence, while it is difficult to be definitive about limiting performances, the examples provided here may be regarded to be what can reasonably be expected of each technique. Since 6 twists appears to be a realistic possibility it may be expected that this will eventually be achieved but that 7 twists in 3 somersaults are unlikely to be realised.

\section{Acknowledgement}

The insights into current performances provided by Daniel Murphy (Canadian National Coach) and Michel Roth (Swiss National Coach) are gratefully acknowledged.

\section{Conflict of Interest Statement}

there are no issues of conflict of interest arising from the personal or professional associations of the author.

\section{REFERENCES}

Chandler, R.F., Clauser, C.E., McConville, J.T., Reynolds, H.M., Young, J.W., 1975. Investigation of inertial properties of the human body. AMRL-TR-74-137, Aerospace Medical Laboratories, WrightPatterson Air Force Base, Ohio. 
Federation Internationale de Ski, 2010. FIS International Freestyle Judging Handbook. http://www.fisski.com/uk/disciplines/freestyle/freestyle-skiing-rules/rules.html

Hiley, M.J., Yeadon, M.R., 2003. Optimum technique for generating angular momentum in accelerated backward giant circles prior to a dismount. Journal of Applied Biomechanics 19, 119-130.

Yeadon, M.R., 1989. Twisting techniques used in freestyle aerial skiing. International Journal of Sport Biomechanics 5, 275-281.

Yeadon, M.R., 1990a. The simulation of aerial movement - III: The determination of the angular momentum of the human body. Journal of Biomechanics 23, 75-83.

Yeadon, M.R., 1990b. The simulation of aerial movement - II. A mathematical inertia model of the human body. Journal of Biomechanics 23, 67-74.

Yeadon, M.R., 1993a. The biomechanics of twisting somersaults. Part I: Rigid body motions. Journal of Sports Sciences 11, $187-198$.

Yeadon, M.R., 1993b. The biomechanics of twisting somersaults. Part II: Contact twist. Journal of Sports Sciences 11, 199-208.

Yeadon, M.R., 1993c. The biomechanics of twisting somersaults. Part III: Aerial twist. Journal of Sports Sciences 11, 209-218.

Yeadon, M.R., Atha, J. and Hales, F.D., 1990. The simulation of aerial movement - IV: A computer simulation model. Journal of Biomechanics, 23, 85-89. 